\title{
An efficient computational approach to balance the trade-off between image forensics and perceptual image quality
}

\author{
Shashidhar T M${ }^{1}$, K B Ramesh ${ }^{2}$ \\ ${ }^{1}$ Department of Electronics and Communication and Engineering, AMC Engineering College, India \\ ${ }^{2}$ Department of Electronics and Instrumentation Engineering, RVCE, India
}

\section{Article Info \\ Article history: \\ Received Jan 7, 2019 \\ Revised Apr 9, 2019 \\ Accepted Apr 17, 2019 \\ Keywords: \\ Digital image processing Image \\ Image forensics \\ Image perceptual quality \\ Un supervised learning}

\begin{abstract}
The increasing trends of image processing applications play a very crucial role in the modern-day information propagation with the ease of cost effectiveness. As image transmission or broadcasting is the simplest form communication which determines easy, fastest and effective way of network resource utilization, thereby since past one decade it has gained significant attention among various research communities. As most of the image attributes often contains visual entities corresponding to any individual, hence, exploration and forging of such attributes with malicious intention often leads to social and personal life violation and also causes intellectual property right violation when social media, matrimonial and business applications are concerned. Although an extensive research effort endeavored pertaining to image forensics in the past, but existing techniques lack effectiveness towards maintaining equilibrium in between both image forensics and image quality assessment performances from computational viewpoint. Addressing this limitation associated with the existing system, this proposed study has come up with a novel solution which achieves higher degree of image forensics without compromising the visual perception of an image. The study formulates an intelligent empirical framework which determines cost-effective authentication of an image object from both complexity and quality viewpoint. Finally, the study also presented a numerical simulation outcome to ensure the performance efficiency of the system.
\end{abstract}

Copyright (c) 2019 Institute of Advanced Engineering and Science. All rights reserved.

\section{Corresponding Author:}

Shashidhar T M,

Research Scholar, Department of Electronics and Communication \& Engineering,

AMC Engineering College,

Bengaluru, India.

Email: shashidhartm2014@gmail.com

\section{INTRODUCTION}

In this era of various social media applications such as Facebook, Twitter etc. along with different cloud based matrimonial and business applications, there is an increasing demand for image attribute exchange. It basically results in a higher degree of vulnerability where image can be maliciously tampered using an editing tool [1-3]. These types of tools are mostly use to manipulate the image content in a way where visual interpretation of subjects pertaining to that particular image become challenging for a viewer. It can be seen that this area of research is more than one decade old, where digital image content privacy preservation problems are at top most concern from authentication viewpoint $[4,5]$. Although a large set of archives emphasized towards identification of image forgery or tampering detection with different forms of research but majority of them do not impose any full-proof solutions that reported as benchmark till date. Moreover, applying image forensics to reveal the underlying fact often leads of collateral loss of image information which is still a gap need to be address. Thereby, in this literature a computational mechanism is 
conceptualized and developed for visual descriptors to extract ROI features from the major block object, probably taking out ROI like vision, or other important constructs in a human face which plays important role to help forensic process as these are the areas which are forged [6-8]. The dimensionality of the image objects is usually very high that requires splitting of the samples. The objective function is to maximize the classification accuracy; therefore, the optimality is iterated between the balance of the ratio of training versus testing dataset to handle trade-off of accuracy and training. The classifying capacity is built on the amount and feature set, training of the amount and feature set training of the training sample set, where as the model validation for its accuracy is done on the test data. The conventional statistical approach as constant to the machine learning approach is developed without the separation and splitting process of the into training and testing $[9,10]$.

The prime focus laid here is to achieve maximum possible accuracy in image forensics operation while compromising the quality of image data. The study also incorporates a computationally efficient image forensic mechanism assisted by un-supervised learning approach which determines accuracy of image object classification and detection. The consecutive segments of this paper are organized as Section 1.2 which basically highlights the exiting state-of-the-art approaches which has also addressed the similar problem and also based on the investigation it identifies and illustrates the problem in Section 1.3. Section 2 basically highlights the empirical design and modeling of the proposed approach followed by elaborated discussion of numerical outcome obtained in Section 3. Finally, Section 4 concludes the contributory aspects of the proposed research work.

This section basically extracts the underlying facts from mostly cited conventional literatures where the prime emphasize has been inclined towards image forensics. The study of Fan et al. [11] presented an optimization-oriented approach which applies approximation to enhance a median filtered image quality but the authors have reported this model to be working with anti-forensics principles. Carvalho et al. [12] explored various transformation-oriented principles to determine image illuminant maps and come up with a novel forensic technique which applies statistical distribution properties to locate the forged region. The authors have claimed that it achieves classification accuracy of $94 \%$ and $84 \%$ respectively. A novel theoretical approach for blind forensics of digital images using geometric transformation is presented in the study of Chen et al. [13]. One the other hand [14] also focused on the same but it basically considers median filtering for digital images. Stamm et al. [15] basically explored the area of anti-forensics and formulate a framework to eliminate compression fingerprints from a digital image, transformation coefficients. Cao et al. [16] focused on the image visual quality enhancement at the same time also targeted to enforce effective image forensics with JPEG compression and pixel value mapping principles. Extensive simulation outcome further claimed its efficiency towards objectifying forged locations. [17-20] also have focused on the similar problem with theoretical as well as experimental discussion. Similarly, Conotter et al. [21] developed a novel forensic technique which utilizes probability of distributions of discrete cosine transformation (DCT) to extract underlying knowledge from image attributes. The study also designs an efficient classifier which can extract significant features from an image object without affecting the quality of the data. Highlights of the study carried out by [22] and [23] also provides an insight into learning approaches for effective decision fusion enabled image forensics. Thai et al. [24] also presents a novel image forensics technique where JPEG quantization plays a crucial role. Murthy et al. [25] have demonstarted a technique. Reddy et al. [26] have demonstarted comparative study of common edge recognization algorithm by using pre-processing method. Kumar and Kishore [27] have presented categorization of Indian classical dance mudra by using HOG characteristic and SVM classifier.

The analysis of the mostly cited exiting literatures clearly reveals the fact that there is still a gap existing when both quality factor and image forensics are concerned. Very few studies are found which completely addresses the problem of image forensics by incorporating image quality enhancement process. It is also observed that most of the existing archives are theoretically illustrated where no benchmarking has been reported with respect to computational, quality and classification accuracy aspects.

The existing image forensic approaches very less likely incorporated machine learning, specifically un-supervised learning based solutions for the detection of forged region which is a prime aspect towards speeding up the process with higher accuracy. Therefore the problem statement in this context can be framed as: "Designing an efficient and intelligent computational model to strengthen the image forensic aspect from both computation and quality of the image view-point is very much challenging" 


\section{EMPIRICAL SYSTEM DESIGN AND MODELING}

\subsection{Process of selection of input data}

This computational process is modeled to select specific file with specific data type (file $e_{\text {dataType }}$ ) from a disk drive location $\left(d_{\mathrm{LoC}}\right)$ by incorporating a filterization mechanism. In the successful file type selection, the process returns two different string which contains $\mathrm{Obj}_{\text {nameand }} \mathrm{Obj}_{\text {path. }}$.

The Table 1 shows selection of a test input data file which is a facial object of .jpg extension. It also shows the outcome of the numerical computing process where values of respective size of the data file with respect to parameters bytes and class are highlighted. On the completion of this numerical computation process the function $f_{\text {ObjSelection }}$ ()returns two different strings such as $\mathrm{Obj}_{\text {name }}[]_{1 \times 10}$ and $\mathrm{Obj}_{\text {path }}[]_{1 \times 52}$ are discussed further.

Table 1. Selection of a Test Obj

\begin{tabular}{ccccc}
\hline Input Data/Obj & file dataType $_{\text {a }}$ extension $)$ & Size on disk & Bytes & Class \\
\hline Test $\mathrm{D}_{\text {obj }}$ & .jpg & $194 \times 259 \times 3$ & 150738 & Uint 8 \\
\hline
\end{tabular}

\subsection{Process of declaring file descriptor $\left(\mathbf{f}_{\mathrm{des}}\right)$}

In this process a string variable named $f_{\text {des }}$ of size $1 \times 16$ is created by concatenating two different string objects $\mathrm{Obj}_{\text {name }}[]_{1 \times 10}$ and $\mathrm{Obj}_{\text {path }}[]_{1 \times 52}$ which generalizes the address of the specific input data which is also can be referred as $d_{\mathrm{LoC}}$.

\subsection{Numerical computation of a data object (Dobj)}

In this process a data object $D_{o b j}$ is created by performing quantization and sampling of a specified data file descriptor $\boldsymbol{D}_{\mathrm{obj}}$. After that a numerical representation of the $D_{o b j}$ is also computed.

\subsection{Detection of major Obj (mObj)}

In this process a user defined function is called where major objects are cropped by invoking a package called vision $v$. $v$ basically detects object using Viola-Jones algorithm. Further it also computes boundary box values $\left(\mathrm{BB}_{\mathrm{v}}\right)$ based on the number of Obj. Further it crops the $\mathrm{D}_{\mathrm{Obj}}$ with respect to the $\mathrm{BB}_{\mathrm{v}}$ defined. The cropped data $\left(\mathrm{mobj}_{\mathrm{j}}\right)$ further saved into a database file $\mathrm{m}_{\mathrm{Obj}}(90 \times 126 \times 3)$ uint 8 .

$$
\left|\vec{m}_{\text {obj }}\right|=\left[\begin{array}{cccc}
m_{11} & m_{12} & \ldots & m_{1 j} \\
m_{21} & m_{22} & \ldots & m_{2 j} \\
\cdot & \cdot & & \cdot \\
\cdot & \cdot & \ldots & \cdot \\
\cdot & \cdot & & \cdot \\
m_{i 1} & m_{i 2} & \ldots & m_{i j}
\end{array}\right]
$$

\subsection{Process of sub object detection from major Obj}

The sub object which is also termed as $\mathrm{Sub}_{\mathrm{obj}} \in \boldsymbol{m o b j e x t r a c t e d ~ w i t h ~ r e s p e c t ~ t o ~ R O I ~ a n d ~ o p t i m i z e d ~}$ output response (OPR). The image forensics here applies a set of computational steps to optimize the performance associated with the major object detection accuracy. The major object detector operates with the significant features and attributes to crop the major object attributes which play a very crucial role while performing the assessment of image forensics.

Finally using the boundary box attributes, the $m_{O b j}$ is extracted with the negligible computational complexity and the cropped region attributes get extracted. The unsupervised learning process in this context uses in-built feature extraction and training mechanism with data pattern followed in visual descriptors to make the classification process much intelligent. The Figure 1 shows a block-based representation to depict the idea of the concept which is imposed in the proposed un-supervised learning-based image forensics methodology while quality assessment of the image also plays a vital role.

The Figure 1 clearly exhibits the block based architectural design of the proposed system. It basically incorporates a functionality which enables the visual descriptors to extract significant features from each block of the data object or $\boldsymbol{m}_{\boldsymbol{O}} \boldsymbol{o b j}_{\mathrm{j}}$. Here each vector basically composed of spatial color information associated with the corresponding blocks. These significant extracted features are used to train the unsupervised classifier to make it more intelligent for getting insight into the underlying attributes of a major object. The training and a testing modeling also introduced here during the conceptualization of the idea. 
If the learning process finds any indication which states that there exists similarity of pattern in between the visual descriptor-oriented vectors, then it reflects that counterfeit is performed to the corresponding block attributes which got similar patterns. During the process operation the color object get converted into 8-bit gray level to optimize the time complexity level prior performing image forensics. The ease of computation basically speeds up the computation at the learning process where the feature extraction and training get performed simultaneously to provide more insight into underlying facts associated with data object and $\boldsymbol{m}_{\boldsymbol{o b j}}$ blocking attributes. Further, the data object detected is processed through a $3 \times 3$ mean filter kernel to make it blur gray level data object at the first step. The visual descriptor methods which extracts the visual properties from the object basically applies a blurring process as there is a reason lies behind. It basically attempts to minimize the negligible variations among the adjacent pixels.

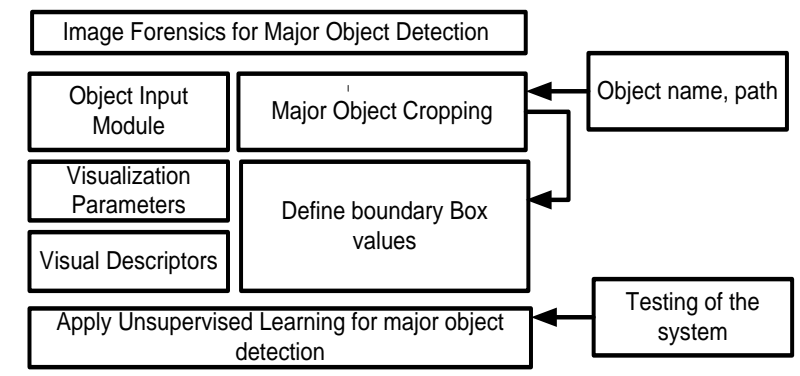

Figure 1. The architectural block-based system overview

Overlapping blocks of size $8 \times 8$ of the blurred image are then used to compute color vectors in visual descriptors. The data object is scanned in row major from left to right and top to bottom. The visual descriptors method is designed to extract features from each block. Pixel values are discretized in order to map intensity into [0 - 63] range. Visual descriptors of the block are determined according to new process. Visual descriptors of each block are of size 128, where first sixty-four elements represent the number of consistency pixels of the corresponding intensity value and last sixty-four elements represent the number of incoherent pixels of the corresponding intensity value in the block. The next section of the study discusses about the outcome obtained after simulating the proposed system in a computing environment for major object detection with cost-effective image forensics.

\section{EXPERIMENTAL ANALYSIS}

This section presents the outcome being accomplished from the extensive numerical simulation which is carried out in 64-bit windows system power by Intel core i5-8250U CPU running with computing speed of $160 \mathrm{GHz} / 1.80 \mathrm{GHz}$. The numerical simulation for the experimental set up also imposes a minimum requirement of 4 GB internal memory (RAM). The extensive comparative analysis carried out with respect to two different prime and significant performance parameters which are i) Peak Signal to Noise Ratio (PSNR) and ii) Execution Time to validate the performance factor of the proposed system as compared to the existing system [21].

The comparative analysis as highlighted clearly shows that the proposed system achieves considerable image forensics while accomplishing higher PSNR values for iteration (10-90) as shown in Figure 2. The peak value of the PSNR obtained at the iteration number 80. The PSNR factor indicates that the proposed technique achieves detection of major objects without compromising the quality factor associated with the tested object during image forensics. It is also found that in the case of existing system the PSNR values are quite lesser.

As shown in the Figure 3, the study also carried out assessment of the time complexity of the proposed system. It shows that the conversion of object from 3-dimensional space to 8-bit 1-dimensional grayscale reduce the dimensionality of data associated with the object which results in optimized computation time. It is also observed that the classifier performs learning and detection of the major object simultaneously with a feature extraction process which also leads to pose negligible computational overhead at the time of simulation. The above quantitative interpretation of from Figure 3 clearly shows that the proposed system accomplishes very lesser computation time as compared to the existing system. The justifications on the basis of numerical simulation outcome clearly shows that the proposed system achieves higher degree of image forensics without affecting the quality aspect associated with the image/object. 


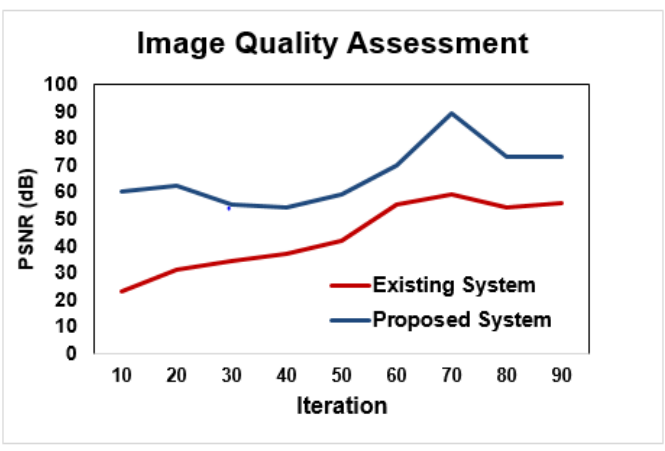

Figure 2. Comparative analysis on the basis of PSNR (dB)
Analysis of Processing Time (Sec)

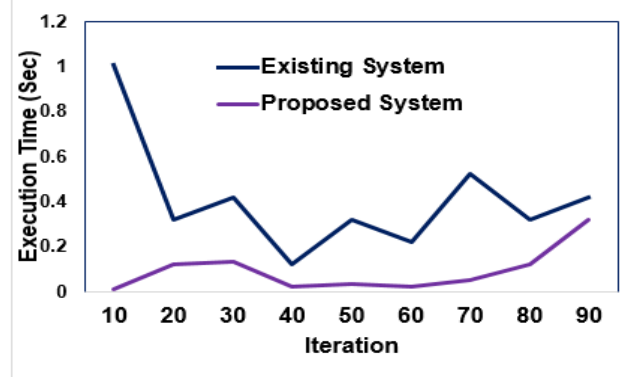

Figure 3. Comparative analysis of execution time $(\mathrm{Sec})$

\section{CONCLUSION}

In the current time there are various research archives which talk about different types of forensic techniques which are found well-capable of detecting forged region as well as artifacts from different image objects. The proposed study presents an analytical form of computational model which imposes higherdegree of image forensics to determine computationally efficient detection of major objects while also balances the quality attributes of the image object. The numerical simulation reveals its efficiency in terms of computational time and quality factor which outperforms the existing baseline with an improvement of almost $50 \%$.

\section{REFERENCES}

[1] A. T. S. Ho and S. Li, "Handbook of Digital Forensics of Multimedia Data and Devices," John Wiley \& Sons, 2015.

[2] H. T. Sencar and N. Memon, "Digital Image Forensics: There is More to a Picture than Meets the Eye," Springer Science \& Business Media, 2012.

[3] R. Pal, "Innovative Research in Attention Modeling and Computer Vision Applications," IGI Global, 2015.

[4] C. Haritha, et al., "A survey on modern trends in ECG noise removal techniques," 2016 International Conference on Circuit, Power and Computing Technologies (ICCPCT), Nagercoil, pp. 1-7, 2016.

[5] Y. Zou, et al., "Automatic Identification of Artifact-related Independent Components for Artifact Removal in EEG Recordings," IEEE J Biomed Health Inform., vol/issue: 20(1), pp.73-81, 2016.

[6] M. Seadle, "Quantifying Research Integrity," Morgan \& Claypool Publishers, 2016.

[7] J. G. R. Elwin, et al., "Survey on passive methods of image tampering detection," 2010 International Conference on Communication and Computational Intelligence (INCOCCI), Erode, pp. 431-436, 2010.

[8] Z. Z. Hua and W. W. Yan, "A lossless compression method of JPEG file based on shuffle algorithm," 2010 2nd International Conference on Advanced Computer Control, Shenyang, pp. 160-162, 2010.

[9] Shashidhar T. M. and K. B. Ramesh, "FARIP: Framework for Artifact Removal for Image Processing Using JPEG," Computer Science On-line Conference. Springer, Cham, 2018.

[10] Shashidhar T. M. and K. B. Ramesh, "Reviewing the Effectivity Factor in Existing Techniques of Image Forensics," International Journal of Electrical and Computer Engineering, vol/issue: 7(6), 2017.

[11] W. Fan, et al., "Median Filtered Image Quality Enhancement and Anti-Forensics via Variational Deconvolution," in IEEE Transactions on Information Forensics and Security, vol/issue: 10(5), pp. 1076-1091, May 2015.

[12] T. Carvalho, et al., "Illuminant-Based Transformed Spaces for Image Forensics," in IEEE Transactions on Information Forensics and Security, vol/issue: 11(4), pp. 720-733, Apr 2016.

[13] C. Chen, et al., "Blind Forensics of Successive Geometric Transformations in Digital Images Using Spectral Method: Theory and Applications," in IEEE Transactions on Image Processing, vol/issue: 26(6), pp. 2811-2824, Jun 2017.

[14] H. Yuan, "Blind Forensics of Median Filtering in Digital Images," in IEEE Transactions on Information Forensics and Security, vol/issue: 6(4), pp. 1335-1345, Dec 2011.

[15] M. C. Stamm and K. J. R. Liu, "Anti-forensics of digital image compression," in IEEE Transactions on Information Forensics and Security, vol/issue: 6(3), pp. 1050-1065, Sep 2011.

[16] G. Cao, et al., "Contrast Enhancement-Based Forensics in Digital Images," in IEEE Transactions on Information Forensics and Security, vol/issue: 9(3), pp. 515-525, Mar 2014.

[17] W. Luo, et al., "JPEG Error Analysis and Its Applications to Digital Image Forensics," in IEEE Transactions on Information Forensics and Security, vol/issue: 5(3), pp. 480-491, Sep 2010.

[18] Y. Hsu and S. Chang, "Camera Response Functions for Image Forensics: An Automatic Algorithm for Splicing Detection," in IEEE Transactions on Information Forensics and Security, vol/issue: 5(4), pp. 816-825, Dec 2010. 
[19] A. A. de Oliveira, et al., "Multiple Parenting Phylogeny Relationships in Digital Images," in IEEE Transactions on Information Forensics and Security, vol/issue: 11(2), pp. 328-343, Feb 2016.

[20] G. Valenzise, et al., "Revealing the Traces of JPEG Compression Anti-Forensics," in IEEE Transactions on Information Forensics and Security, vol/issue: 8(2), pp. 335-349, Feb 2013.

[21] V. Conotter, et al., "Forensic Detection of Processing Operator Chains: Recovering the History of Filtered JPEG Images," in IEEE Transactions on Information Forensics and Security, vol/issue: 10(11), pp. 2257-2269, 2015.

[22] E. Ardizzone, et al., "Copy-Move Forgery Detection by Matching Triangles of Keypoints," in IEEE Transactions on Information Forensics and Security, vol/issue: 10(10), pp. 2084-2094, Oct 2015.

[23] M. Fontani, et al., "A Framework for Decision Fusion in Image Forensics Based on Dempster-Shafer Theory of Evidence," in IEEE Transactions on Information Forensics and Security, vol/issue: 8(4), pp. 593-607, Apr 2013.

[24] T. H. Thai, et al., "JPEG Quantization Step Estimation and Its Applications to Digital Image Forensics," in IEEE Transactions on Information Forensics and Security, vol/issue: 12(1), pp. 123-133, Jan 2017.

[25] G. S. N. Murthy and T. Veerraju, "A novel approach based on decreased dimension and reduced gray level range matrix features for stone texture classification," International Journal of Electrical and Computer Engineering, vol/issue: 7(5), pp. 2502, 2017.

[26] Reddy, et al., "Comparative Analysis of common Edge Detection Algorithms using Pre-processing Technique," Int. J. Electr. Comput. Eng (IJECE), vol/issue: 7(5), pp. 2574-2580, 2017.

[27] K. V. V. Kumar and P. V. V. Kishore, "Indian Classical Dance Mudra Classification Using HOG Features and SVM Classifier," International Journal of Electrical \& Computer Engineering, vol/issue: 7(5), 2017.

\section{BIOGRAPHIES OF AUTHORS}

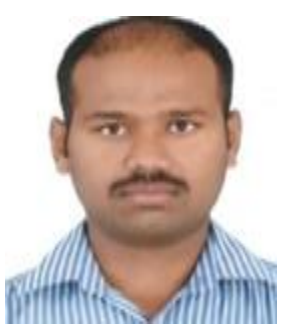

Shashidhar T M., Research Scholar, Visvesvaraya Technological University Belagavi, Karnataka, India. Currently pursuing PhD under RVCE (VTU), Karnataka, India. His teaching experience is around 11 years. Hisresearch area is Signal Processing. He has completed hisM. Tech (Digital electronics and communication system) from PESIT, Bengaluru, Karnataka, India. Alsocompleted B.E. (Electronics and communication), from SJMIT, Chiradurga, Karnataka, India.

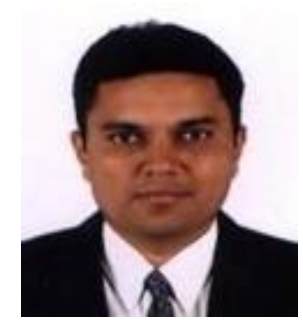

K.B. Ramesh, Associate Professor and Head, Department of Electronics and Instrumentation Engg.R V college of Engineering, Bengaluru, Karnataka, India. Hehas completed $\mathrm{PhD}$ in Computer Science and Engineering from Kuvempu University. He hasaround twenty-threeyears (23) of teaching experience in E\&I Engg. His major research area is in Computer Science and Engineering and minorresearch area is in Biomedical Engineering/Bioinformatics. 